\title{
Regression Modelling and Analysis of Cell phone-Based Syndromic Surveillance Data for Ebola in Sierra Leone
}

\section{Jia Bainga Kangbai*}

Department of Environmental Health Sciences, School of Community Health Sciences, Njala University, Bo Campus, Sierra Leone

*Corresponding author: Jia Bainga Kangbai, Department of Environmental Health Sciences, School of Community Health Sciences, Njala University, Bo Campus, Sierra Leone, E-mail: jiakangbai@hotmail.com

Received date: June 7, 2016; Accepted date: June 16, 2016; Publication date: June 22, 2016

Copyright: ( 2016 Kangbai JB. This is an open-access article distributed under the terms of the Creative Commons Attribution License; which permits unrestricted use; distribution; and reproduction in any medium; provided the original author and source are credited.

\begin{abstract}
Aim: To determine the out degree centrality for multivariate analysis, examine the demographic differences and characteristics of callers who make more calls, and determine callers who are more likely to make at least one positive Ebola call.
\end{abstract}

Methods: Surveillance data for 393 suspected Ebola cases (192 males, 201 females) were collected from October 23, 2014 to June 28, 2015 using cell phone technology. UCINET and Net Draw software were used to determine the out degree centrality used for multivariate analysis. Poisson and logistic regression analyses were used to do multivariable analysis.

Result: Women $(\mathrm{AOR}=0.33,95 \% \mathrm{Cl}[0.14,0.81])$ was associated with decreased odds of making at least one positive Ebola surveillance call compared to men. Women (IR=0.63, 95\% Cl $[0.49,0.82]$ ) was also associated with making fewer Ebola surveillance calls compared to men.

Conclusion: This study shows that the combination of cell phone technology with user-friendly and open-source social network software such as UCINET and Net draw will provide an important adjunct to the traditional measures of epidemiologic surveillance.

Keywords: Ebola; Syndromic; Surveillance; Centrality; Analysis; Cell phone; Out degree

\section{Introduction}

Ebola virus disease (EVD) was first identified in 1976 in Zaire but there has been more cases of the disease in other countries in recent times [1]. EVD is characterised by febrile illnesses and is naturally transmitted by either animal or vector hosts [2]. The 2014 West African Ebola outbreak is caused by Ebola virus belonging to the hemorrhagic fever viruses (HFVs) family Filoviradae. There are 5 different virus strains that causes ebola disease: Sudan virus, Tai Forest virus, Reston virus, Ebola virus, and Bundibugyo virus. The 2014 Ebola outbreak is the largest so far and recorded more than 20,000 cases and 10,000 deaths [3]. Sierra Leone was one of the countries greatly affected by the 2014 West Africa Ebola outbreak [4].

Sierra Leone's first Ebola case was confirmed on 27 $7^{\text {th }}$ May 2014 Prior to 2014 Sierra Leone's preparedness for an EVD outbreak was lacking. Sierra Leone's EVD response involved the formation of an Ebola technical task force that was responsible for EVD surveillance, case identification, case tracking and monitoring. Contact tracing, Ebola case identification, treatment, management, and the effective response to both patients and the community have been shown to be effective for EVD surveillance in the past [5].

Correct coordination between Ebola case isolation and treatment, contact tracing and follow-up of contact for 21 days after exposure was very effective in controlling the spread of Ebola outbreak in West
Africa in 2014 [6]. Sierra Leone embarked on using cell phone technology to assist with its EVD surveillance in 2014.

Cell phone technology has revolutionized disease surveillance by serving as a channel through which people reveal their public health concerns, locations, and movements. In Haiti, public health experts successfully predicted the spatial evolution of the 2010 cholera outbreak using cell phone calls and SMS messages obtained from more than 2 million mobile phone SIM cards [7].

Following the 2014 Ebola outbreak, researchers in West Africa called for the use of routine syndromic surveillance systems that rely on data supplied via cell phones. Researchers also recommended the use of cell phones for relaying surveillance data from communities affected by an EVD outbreak to peripheral health centres [8].

In Guinea, smartphones were used to communicate real-time surveillance data for contact tracing and case identification during the 2014 West Africa EVD outbreak [9]. Given the surge in global cell phone usage and the increasing popularity of cell phone-based epidemiological surveillance more research is needed to explore its efficiency and community uptake. Network showing connectivity of callers who made multiple calls and suspected Ebola cases for Moyamba District from October 2014 to June 2015 in Figure 1.

Furthermore, few studies have taken advantage of the network-like data generated from cell phone-based surveillance in which callers and cases are interconnected through a web of calls. 


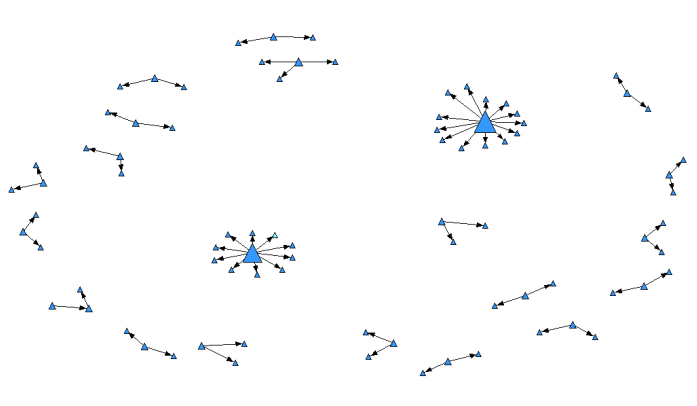

Figure 1: Network showing connectivity of callers who made multiple calls and suspected Ebola cases for Moyamba District from October 2014 to June $2015 .{ }^{*}$ Arrows showing outgoing surveillance calls to positive and suspected Ebola cases.

This study used social network analysis to evaluate Ebola surveillance data collected in Sierra Leone using cell phone technology. Specifically this study (1) used UCINET and Netdraw software to determine out degree centrality which was subsequently used in the Poisson regression (2) examined the demographic differences in surveillance callers who used the cell phone surveillance system; and (3) determined the characteristics of callers who were more likely to make at least one positive Ebola surveillance call (i.e., those who called about suspected cases that were subsequently confirmed to be Ebola positive).

\section{Material and Methods}

\section{Study setting and participants}

Surveillance data were collected from Moyamba District in southern Sierra Leone from October 2014 to June 2015 using cell phones. The district is approximately $6,902 \mathrm{sq} / \mathrm{km}$ with a population of 258,506 9 (Sierra Leone 2004 Population Census, 2014). In 2014 the population of Sierra Leone was estimated to be 6, 315, 627 of which approximately 4,215,000 (70\%) had cell phones (Global Resources and Information Directory, 2015). The Moyamba District Health Manager Team (MDHMT) Ebola Taskforce, in collaboration with Action Contra la famine (ACF), started cell phone-based syndromic surveillance for Ebola in October 2014. Specifically, Moyamba District community members will call an Ebola hotline and provide their names, telephone number, and the village in which they were residing. The caller also provided the name of the person they suspected to have Ebola and the person's sex, age and the village of the person that they suspected to have Ebola. Within 24 hours of receiving a call, the MDHMT Ebola Taskforce would dispatch a Community Health Officer and Ebola contact tracers to the location of the person suspected to have Ebola and transfer him/her to an Ebola treatment centre for diagnosis. If the suspected Ebola case was deceased his/her body was transferred to the local mortuary for safe burial. Deceased persons however were not tested for Ebola.

The dataset for analysis in this study included callers' names, their telephone numbers, and their village of residence, as well as the cases' name, sex, and location (village), whether the person was sick or dead at the time the surveillance call was made, Ebola lab result for suspected Ebola cases, and if there were sick or dead people at the residence of the suspected Ebola case at the time the call was made to the MDHMT call center. The names and demographic characteristics of callers and cases were cross-referenced to produce a network between callers and cases covering the entire surveillance period. The study used UCINET and Netdraw software. Outdegree centrality is the number of ties or contact a node has within a network [10].

\section{Analysis}

SAS 9.2 version [11] was used for descriptive and summary statistical analysis of the characteristics of the callers and suspected Ebola cases. Poisson regression was used to determine the gender difference in the number of Ebola surveillance calls made controlling in the model for the week in which the surveillance call was made, and the Ebola prevalence of the village in which calls were made. Logistic regression was used to determine the gender difference associated with making at least one positive Ebola surveillance call, controlling in the model for the week in which Ebola surveillance calls were made, whether the person for whom the Ebola surveillance call was made was sick or dead, and the Ebola prevalence of the village in which calls were made.

\section{Ethics and privacy}

The University of Kentucky Institutional Review Board reviewed the protocol for the secondary analyses described in this capstone and determined that it met federal criteria to be exempt.

\section{Results}

\section{Descriptive characteristics of suspected ebola cases}

Surveillance data for 393 suspected Ebola cases (192 males, 201 females) were collected from October 23, 2014 to June 28, 2015 using cell phone technology. The descriptive characteristics of suspected Ebola cases, callers, and status of Ebola suspected cases, lab results and type of call are presented in (Table 1).

The average age of the suspected Ebola cases was 23.5 years (standard deviation=29.5). Three hundred and twenty-four $(82.4 \%)$ of the suspected Ebola cases were reported sick at the time data was collected while $69(17.6 \%)$ were deceased.

Two hundred and twenty (68\%) of the sick suspected Ebola cases were females while 104 (32\%) were males. Twenty-five (8\%) of the sick suspected Ebola cases were later confirmed as infected with Ebola, 295 (91\%) were tested negative for Ebola while $4(1 \%)$ had unknown infections.

\begin{tabular}{|l|l|}
\hline Characteristics & $\mathbf{n}(\%)$ \\
\hline Suspected Cases $(n=393)$ & \\
\hline Sex & \\
\hline Male & $192(48.9)$ \\
\hline Female & $201(51.2)$ \\
\hline Status & \\
\hline Sick & $324(82.4)$ \\
\hline Dead & $69(17.6)$ \\
\hline
\end{tabular}


Page 3 of 5

\begin{tabular}{|l|l|}
\hline Lab result (among 324 sick suspected Ebola cases) & \\
\hline Positive & $25(8)$ \\
\hline Negative & $295(91)$ \\
\hline Unknown & $4(1)$ \\
\hline Village Ebola prevalence - median (interquartile range) & $0(0)$ \\
\hline Callers (n=353) & \\
\hline Sex & \\
\hline Male & $301(85.3)$ \\
\hline Female & $52(14.7)$ \\
\hline Number of calls per person - median (interquartile range) & $1(0)$ \\
\hline One call & $339(93.9)$ \\
\hline Two or more calls & $54(6.1)$ \\
\hline
\end{tabular}

Table 1: Descriptive characteristics and status of suspected Ebola cases and callers, lab result and type of call.

\section{Descriptive characteristics of surveillance callers}

Three hundred and fifty-three callers (males $=301$, females $=52$ ) reported syndromic surveillance data for Ebola for the period under review. Three hundred and thirty-six (95.2\%) callers made single call while seventeen (4.8\%) made multiple calls. Of the multiple callers; 14 made two calls, one made 3 calls, one made 10 calls, and another made 13 calls.

Twenty-two (2 females and 20 males) caller reported suspected Ebola cases with $100 \%$ efficiency (i.e., all the calls made were later confirmed to be Ebola positive). Three male callers made two calls each with one call resulting in one confirmed Ebola case (i.e. 50\% efficiency). All other callers had $0 \%$ efficiency (i.e., every suspected Ebola case about whom they called tested negative).

\section{Descriptive characteristics of calls}

The distribution of calls by gender is represented in (Table 2). Men made fewer (16.7\%) surveillance calls about dead suspected Ebola cases compared to women (17.3\%). Majority (83.3\%) of the calls made by men were about sick suspected Ebola cases.

The percentage of men making at least one positive Ebola surveillance call was nearly twice $(6.8 \%)$ as high than that for women (3.7\%).

\begin{tabular}{|l|l|l|}
\hline Characteristics & Male & Female \\
\hline Number of calls & 341 & 52 \\
\hline Number of positive calls & 23 & 2 \\
\hline Call Status of suspected cases - n(\%) & & \\
\hline Sick & $284(83.3)$ & $43(82.7)$ \\
\hline Dead & $57(16.7)$ & $9(17.3)$ \\
\hline $\begin{array}{l}\text { Lab result (for } 327 \text { sick suspected Ebola } \\
\text { reported calls) }\end{array}$ & & \\
\hline
\end{tabular}

\begin{tabular}{|l|l|l|}
\hline Positive & $23(8.1)$ & $2(4.7)$ \\
\hline Negative & $261(91.9)$ & $41(95.3)$ \\
\hline Number of dead suspected cases not tested & $57(16.7)$ & $9(17.3)$ \\
\hline Number who made at least one positive call & $23(6.8)$ & $2(3.7)$ \\
\hline
\end{tabular}

Table 2: Distribution of status of suspected cases, lab results, number and percentages of positive and negative calls by gender of callers.

\section{Factors associated with number of calls}

Poisson regression results shows gender difference in the number of Ebola surveillance calls made (Table 3). Men made more Ebola surveillance calls than women $(\mathrm{p}<0.01)$. In multivariable analysis, adjusting for other variables in the model, women $(\mathrm{IR}=0.63,95 \% \mathrm{CI}$ $[0.49,0.82])$ were associated with decreased incidence of making Ebola surveillance calls than men. The goodness-of-fit chi-squared test for the Poisson regression was $420.690(\mathrm{df}=389, \mathrm{p}=0.13)$ which indicates that the regression model fits the study data well.

\begin{tabular}{|l|l|l|l|}
\hline Characteristic & IR & $\mathbf{9 5 \%} \mathbf{C l}$ & $\mathbf{p}$-value \\
\hline $\begin{array}{l}\text { Female versus male } \\
\text { gender }\end{array}$ & 0.63 & $(0.49,0.82)$ & $<0.01^{*}$ \\
\hline Village Ebola prevalence & 0.95 & $(0.88,1.02)$ & 0.17 \\
\hline Week & 0.9 & $(0.89,0.91)$ & $<0.0001^{*}$ \\
\hline
\end{tabular}

$\mathrm{IR}=$ Incidence ratio; $\mathrm{Cl}=$ Confidence interval ${ }^{*} \mathrm{p}<0.05$

Table 3: Multivariate analysis of factors associated with the number of Ebola surveillance calls.

\section{Factors associated with making at least one positive call}

Logistic regression results shows gender difference in the likelihood of making at least one positive Ebola surveillance call (Table 4). Women were less likely to make at least one Ebola positive call compared to men $(\mathrm{p}=0.01)$. Holding other covariates in the model constant, the odds ratio estimate for the gender difference (reference group: males) is 0.33 (95\% CI $[0.14,0.81])$. This indicates that women were significantly less likely to make at least one positive Ebola surveillance call than men holding other covariates in the model constant (95\% CI $[0.14,0.81])$.

\begin{tabular}{|l|l|l|l|}
\hline Characteristic & $\begin{array}{l}\text { Adjusted } \\
\text { OR }\end{array}$ & $\mathbf{9 5 \%}$ Cl & p-value \\
\hline $\begin{array}{l}\text { Female versus male } \\
\text { gender }\end{array}$ & 0.33 & $(0.14,0.81)$ & $0.01^{*}$ \\
\hline $\begin{array}{l}\text { Week (1 unit change in } \\
\text { week) }\end{array}$ & 1.05 & $(0.96,1.15)$ & 0.31 \\
\hline Sick versus dead status & 0.52 & $(0.17,1.54)$ & 0.24 \\
\hline OR= Odds ratio; Cl=Confidence interval * $p<0.05$ & \\
\hline
\end{tabular}

Table 4: Multivariate analysis of factors associated with making at least one positive Ebola surveillance call. 
Page 4 of 5

\section{Discussion}

Ebola syndromic surveillance data was collected by 353 callers using cell phone technology in Sierra Leone for the review period. Adjusting the other covariates in the model, women made fewer Ebola surveillance calls than men (IR=0.63, 95\% CI $[0.49,0.82]$ ). From multivariable analysis, women were significantly less likely to make at least one positive Ebola surveillance call than men holding other covariates in the model constant $(\mathrm{AOR}=0.33,95 \% \mathrm{CI}[0.14,0.81])$.

To my knowledge no study has investigated the association between gender, number of cell phone surveillance calls made during an Ebola outbreak, and the likelihood of making at least one positive surveillance call. The reasons for men in Sierra Leone making more surveillance calls than women may be attributed to the higher gender gap in literacy. Forty-five percent of men in Sierra Leone are educated compared to $26 \%$ for women [12]. This higher gender literacy gap makes men more likely to be employed than women. Sierra Leonean women like their counterparts in Guinea and Liberia experience significant gender-based discrimination in terms of employment and education [13]. This gender-based difference in terms of employment and education also means high property-ownership gap between men and women in Sierra Leone. Sierra Leonean women are $43 \%$ less likely than their men to own house, television, computers and a cell phone [14]. Because of these reasons men Sierra Leone were able to make more surveillance calls than women.

Men in Sierra Leone were more likely to make at least one positive surveillance call than women probably due to their increased ability to accurately identified signs and symptoms of Ebola than women. This advantage by men may have been acquired from years of academic and practical clinical training. There are almost twice as many educated men than women in Sierra Leone [12]. Studies have shown that greater experience obtained from years of academic training and clinical practice is associated with post predictive value accuracy in syndromic surveillance. In syndromic surveillance, post predictive value or positive predictive value is the probability of disease given a positive syndromic surveillance test.

This study used few variables for multivariable analysis because surveillance officers were not provided sufficient or complete information by surveillance callers. It may also be possible that MDHMT Ebola surveillance team officers may have incorrectly recorded the names of the callers, suspected Ebola cases or both. It could also be possible that there were some confounders that were unmeasured and hence unaccounted for in the final multivariable models.

In spite of these assumed limitations, health policy and decision makers should not be dissuaded from employing the use of cell phone technology to assist with outbreak surveillance and investigation during infectious disease outbreak [15]. This study shows that the combination of cell phone technology with user-friendly and opensource social network software such as UCINET will provide an important adjunct to the traditional measures of epidemiologic surveillance. The ability to collect surveillance data using cell phone technology during disease outbreak in near or real time may greatly help to decide the best use of disease control resources. This study underscores the need to develop, improve and utilize available tools for outbreak investigation and control.

\section{Conflict of Interest Statement}

There is no conflict of interest attached to this manuscript.

\section{Acknowledgement}

Many thanks to Dr. April Young, Dr. Erin Abner, Dr. W. Jay Christian and Prof. Wayne T. Sanderson- of the Department of Epidemiology in the College of Public Health at the University of Kentucky for proof reading, and providing guidance during this study. Thanks goes also to the Mr. Mohamed Kallon of Action Contra la famin in Moyamba District in Sierra Leone for providing the Ebola surveillance data that was used in this study.

\section{Conclusion}

TINU syndrome is a rare entity with unknown pathogenesis, its association with autoimmune diseases suggests its autoimmune origin. TINU syndrome is secondary to immunological disorders as evidenced interstitial infiltrate of the renal parenchyma; the inflammatory disease of the uvea and good response to corticosteroid therapy. Some diseases have similar findings and can be confused with TINU syndrome, making accurate diagnosis difficult in the absence of characteristic involvement of other organs. Early diagnosis of TINU syndrome is challenging in patients with non-specific symptoms and laboratory findings. It should be emphasized that it is important to pay attention to ocular signs such as eye pain or redness. In addition, measurement of UB2M is essential for early diagnosis of TINU syndrome.

\section{References}

1. Sarwar UN, Sitar S, Ledgerwood (2011) Filovirus emergence and vaccine development: a perspective for health care practitioners in travel medicine. Travel Med Infect Dis 9: 126-134.

2. Hong JE, Hong KJ, Choi WY, Lee WJ, Choi YH, et al. (2014) Ebola hemorrhagic fever and the current state of vaccine development. Osong Public Health Res Perspect 5: 378-382.

3. Zawilińska B, Kosz-Vnenchak M (2014) General introduction into the Ebola virus biology and disease. Folia Med Cracov 54: 57-65.

4. Dallatomasina S, Crestani R, Sylvester Squire J, Declerk H, Caleo GM, et al. (2015) Ebola outbreak in rural West Africa: epidemiology, clinical features and outcomes. Trop Med Int Health 20: 448-454.

5. Tambo E, Xiao-Nong Z (2014) Acquired immunity and asymptomatic reservoir impact on frontline and airport ebola outbreak syndromic surveillance and response. Infect Dis Poverty 3: 41.

6. Pandey A, Atkins KE, Medlock J, Wenzel N, Townsend JP, et al. (2014) Strategies for containing Ebola in West Africa. Science 346: 991-995.

7. Bengtsson L, Gaudart J, Lu X, Moore S, Wetter E, et al. (2015) Using mobile phone data to predict the spatial spread of cholera. Sci Rep 5: 8923.

8. Ansumana R, Bonwitt J, Stenger DA, Jacobsen KH (2014) Ebola in Sierra Leone: a call for action. Lancet 384: 303.

9. Sacks JA, Zehe E, Redick C, Bah A, Cowger K, et al. (2015) Introduction of Mobile Health Tools to Support Ebola Surveillance and Contact Tracing in Guinea. Glob Health Sci Pract 3: 646-659.

10. Borgatti SP, Everett MG, Freeman LC (2002) Ucinet for Windows: Software for Social Network Analysis.

11. SAS (2011) SAS 9.2.3 Help and Docmuentation. SAS.

12. Sierra Leone (2008) Demographic and Health Survey.

13. Bhandari M (2011) Using technology to close the gender gap in Sierra Leone. The Guardian. 
Citation: Kangbai BJ (2016) Regression Modelling and Analysis of Cell phone-Based Syndromic Surveillance Data for Ebola in Sierra Leone. J Trop Dis 4: 214. doi:10.4172/2329-891X.1000214

Page 5 of 5

14. Tambo E, Xiao Nong Z (2014) Acquired immunity and asymptomatic reservoir impact on frontline and airport ebola outbreak syndromic surveillance and response. Infect Dis Poverty 3: 41

15. Manivanna A (2015) Gender inequalities in access to information about Ebola as gender based violence. Harvard Human Rights Journal.
16. (2015) West Africa: Respect Rights in Ebola Response. Human Rights Watch. 